\title{
COMPONENTES DE RENDIMIENTO EN Coffea arabica L. EN TRES ZONAS ALTITUDINALES DEL SUR DE COLOMBIA
}

\section{YIELD COMPONENTS IN Coffea arabica L. IN THREE ALTITUDINAL ZONES OF SOUTHERN COLOMBIA}

\author{
Paula Yuliza Burbano R. • paulaburbano@udenar.edu.co \\ Estudiante Ingeniería Agronómica, Universidad de Nariño, Pasto-Colombia.
}

Angie Lorena Valencia A. • angie.vale19@udenar.edu.co, Estudiante Ingeniería Agronómica, Universidad de Nariño, Pasto-Colombia.

Tulio César Lagos-Burbano•tclagosb@udenar.edu.co

Profesor Titular. I.A., Ph.D. Facultad de Ciencias Agrícolas. Universidad de Nariño. San Juan de Pasto-Colombia.

Citación: Burbano, P., Valencia, A., Lagos-Burbano, T. (2022). Componentes de rendimiento en Coffea arabica L. en tres zonas altitudinales del sur de Colombia. Revista de Investigación Agraria y Ambiental, 13(1), 51 - 62. DOI: https://doi.org/10.22490/21456453.4350

\section{RESUMEN}

Contextualización: El café se cultiva en el $64 \%$ de los municipios de Nariño, donde la variabilidad climática es adecuada para que la planta pueda realizar sus actividades metabólicas.

Vacío de conocimiento: El desconocimiento y la falta de estudios relacionados con la respuesta de los componentes de rendimiento, en diferentes ambientes, dificulta interpretar las variables que influyen en el rendimiento del café variedad Castillo.

Propósito: El objetivo fue establecer la relación causa-efecto entre variables relacionadas con los componentes morfológicos, fisiológicos y climáticos del rendimiento del café, variedad Castillo, considerando tres condiciones altitudinales (Alta-A $=2.015 \mathrm{msnm}$, Media-M $=1.700$ msnmy Baja-B= 1.536 msnm).

Metodología: El trabajo se realizó en el municipio de Sandoná, departamento de Nariño. Las variables registradas incluyeron: radiación fotosintéticamente activa, temperatura ambiente, precipitación, humedad relativa, altura de planta, número de hojas, diámetro basal del tallo, número de ramas primarias, longitud de ramas primarias, número de entrenudos por rama, largo de la hoja, área foliar e índice de área foliar. Estas se evaluaron a través del Análisis de Sendero.

Resultados y conclusiones: LOS componentes de rendimiento en el cultivo de café de la variedad Castillo variaron con la altitud. Las zonas M y B obtuvieron los rendimientos más altos. Para la altitud M (1.700msnm), los efectos directos sobre el rendimiento (RTO) los tuvo la variable ramas primarias (RP). Dentro de los efectos indirectos, los más importantes fueron los causados por humedad relativa $(\mathrm{RH})$ a través de la temperatura $(T)$. La altitud $B$ (1.536 msnm) presentó efectos directos en el largo de rama (LR), área foliar (AF) y Radiación Fotosintéticamente Activa (RFA); y los efectos indirectos estuvieron en la $T$ a través de la RFA, el número de entrenudos (NE) a través de la altura de planta (AP), el diámetro basal (DB) a través del número de hojas (NH), el RP a través de la longitud de la hoja (LH), AF y HR. La zona con menor rendimiento fue la altitud $A(2.015 \mathrm{msnm})$, mostrando como efectos directos AP, NE y $\mathrm{HR}$; y los indirectos, NH, DB, LR y AF a través de AP. Es necesario estudiar la arquitectura de la planta y su relación con la HR para potenciar el RTO del café.

Palabras clave: Altitud; variables climáticas; variables fsiológicas; Análisis de Sendero 


\section{ABSTRACT}

Contextualization: Coffee is cultivated in $64 \%$ of Nariño municipalities, where climate variability is suitable for the plant to carry out its metabolic activities.

Knowledge Gap: The lack of knowledge and the lack of studies related to the response of performance components, in different environments, difficult to interpret which variables influence in the performance of the Castillo variety coffee.

Purpose: The goal was to establish causeeffect relations between variables related to morphological, physiological, and climatic components, with the yield of coffee, Castillo variety, under three altitude ranges (High2.015 meters above sea level, Medium-1.700 meters above sea level and Low-1.536 meters above sea level).

Methodology: The work was carried out in the municipality of Sandoná, Nariño department. The evaluated variables included: Photosynthetically Active Radiation, Ambient Temperature, Precipitation, Relative Humidity, Plant Height, Number of Leaves, Basal Stem Diameter, number of primary branches, length of primary branches, number of internodes per branch, Leaf length, and foliar area index. These were evaluated through the Path Analysis.

Results and conclusions: The performance components in Castillo variety coffee, varied according to altitude. Zones $M$ and $B$ achieved the highest yields. For altitude M (1.700 meters above sea level), the direct effect on the yield $(Y)$ were caused by the primary branches (PB). Within the indirect effects, the most important were those caused by relative Humidity $(\mathrm{RH})$ through temperature $(T)$. Altitude B (1.536 meters above sea level) has direct effects on the branch length (BL), foliar area (FA), and Photosynthetically Active Radiation (RFA); and the indirect effects were $T$ through the RFA, number of internodes (NI) through plant height $(\mathrm{PH})$, basal diameter (BD) through leaf numbers (LN), RP through Leaf Length (LL), AF and HR. The area with the lowest yield was altitude $A$ (2.015 meters above sea level), showing as direct effects the $A P, N E$, and $H R$; the indirect effects were $\mathrm{NH}, \mathrm{DB}, \mathrm{BL}$, and FA through $\mathrm{PH}$. It is necessary to study the architecture of the plant and its relation with HR to enhance the RTO of coffee.

Keywords: Altitude; climatic variables; physiological variables; Path Analysis 


\section{RESUMEN GRÁFICO}

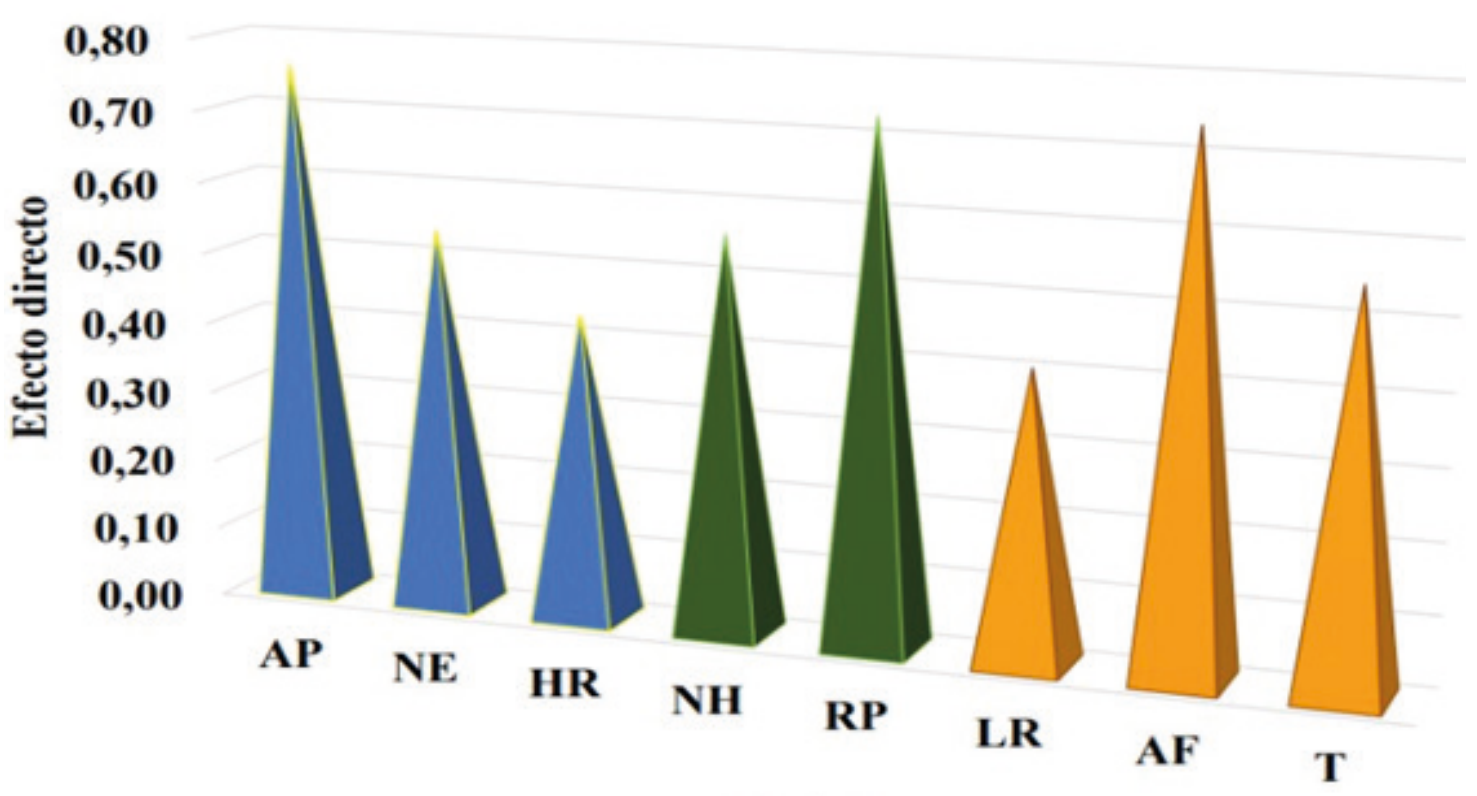

Variable

$2015 \mathrm{msnm}$

$1700 \mathrm{msnm}$

$1536 \mathrm{msnm}$

Variables que registraron los mayores efectos directos sobre el rendimiento de café (Cofea arabica L.) variedad Castillo, en las tres altitudes de estudio localizadas en el municipio de Sandoná, departamento de Nariño.

Fuente: autores.

\section{INTRODUCCIÓN}

En el mundo se producen alrededor de 7,7 millones de toneladas de café pergamino al año, el $85 \%$ se produce en Latinoamérica y el promedio del rendimiento mundial se estima en 0,7 t/ha (Organización Internacional del Café [OIC], 2019). Los países como Colombia, Brasil, Vietnam y Costa Rica tienen promedios superiores al promedio mundial, el cual oscila entre 1-1,4 t.ha-1(Clavijo, 2019). Colombia tiene zonas cafeteras ubicadas en la región Andina Tropical, donde existe gran variabilidad climática dada por el relieve y la altitud, la cual influye directamente sobre la temperatura, la radiación solar, la humedad relativa y la precipitación (Ramírez et al., 2012). Estas variables, están relacionadas directamente con el crecimiento y el desarrollo de las plantas, con la presencia o ausencia de problemas fitosanitarios, con la producción, el rendimiento y la calidad de taza del café (Arcila et al., 2007). En Colombia, las regiones cafeteras se extienden a lo largo del relieve Andino entre 1.000 a 2.000 msnm, ya que en este rango se encuentran las condiciones edafoclimáticas aptas para el cultivo (Jaramillo-Robledo, 2005). El aumento de altitud y la disminución de la temperatura son factores que favorecen la prolongación del proceso de llenado, maduración y peso del grano, así como la producción y la calidad de la bebida (Suárez et al., 2015).

Por otra parte, la competitividad del café del sur del departamento, frente a los mercados nacionales e internacionales, se ve afectado porque se condiciona de manera negativa el factor de rendimiento al momento de la comercialización, impidiendo obtener un valor agregado (Comité departamental de Cafeteros de Nariño [CCN], 2008). El rendimiento del café en Colombia, para el 2019, llegó a 21,40 (sacos de 60 kg) (Ministerio de Agricultura, 2020), mientras que en Nariño el rendimiento fue de 1,09 t.ha-1, superior al promedio nacional $\left(1,06\right.$ t.ha $^{-1}$ ) (Criollo et 
al., 2019). El rendimiento está relacionado con la capacidad fotosintética de la planta, en la que la Radiación Fotosintéticamente Activa (RFA) se convierte en la principal limitante en zonas con sombrío mayor al $40 \%$, y, por consiguiente, las plantas compiten por luz y agua (Zapata et al., 2017). Es importante tener en cuenta que la cantidad de horas luz es un factor importante para la fotosíntesis en el cultivo de café, debido a que éste acelera o disminuye el desarrollo fisiológico de la planta. El brillo solar y la nubosidad están relacionados con la temperatura de una zona, y esta es determinante para los procesos fisiológicos de la planta, dado que actúa como un factor catalizador (Muschler, 1999).

El rendimiento y los componentes de rendimiento dependen básicamente de la expresión de la variedad o genotipo, del ambiente y de la interacción del genotipo por el ambiente (Tirado et al., 2018). Para entender las relaciones que existen entre el genotipo y el ambiente, es necesario conocer la fisiología del cultivo y su relación con las características que definen el rendimiento (Wardofa et al., 2019). Con relación a esto, varios estudios han demostrado que las variables climáticas son los principales factores que condicionan el crecimiento y el buen desarrollo del cultivo de café (Arcila et al., 2007). Sin embargo, Checa y Noguera (2017) obtuvieron que los componentes climáticos no muestran una relación estrecha con los componentes de crecimiento evaluados, debido a que existen otros factores como el tipo de suelo y la disponibilidad mineral que pueden ser determinantes en el cultivo.

El estudio de los componentes de rendimiento en el cultivo de café es una herramienta muy importante en la planificación de los cultivos porque permite estimar la producción a futuro, los costos requeridos para la cosecha, el beneficio, las labores inherentes a la producción y proponer nuevas técnicas agronómicas orientadas al mejoramiento de aquellos factores 0 componentes que presenten mayor incidencia en la producción (Castro et al., 2011). Por esta razón, el objetivo de este trabajo fue identificar la relación entre los componentes morfológicos, fisiológicos y climáticos del rendimiento del café variedad Castillo en tres altitudes del municipio de Sandoná, departamento de Nariño.

\section{MATERIALES Y MÉTODOS \\ Localización}

El presente trabajo se realizó en tres zonas altitudinales (Alta, Media y Baja) del municipio de Sandoná (Tabla 1), el cual tiene una extensión aproximada de $101 \mathrm{Km}^{2}$, una altitud media de $1.817 \mathrm{msnm}$, temperatura promedia de $18^{\circ} \mathrm{C}$, precipitación promedia de $1.091 \mathrm{~mm}$ año-1 y una humedad relativa de $80 \%$ en promedio. Los suelos presentan características homogéneas en los que sobresale su textura limosa, y la zona de vida se encuentra dentro de las categorías bosque húmedo montano bajo (bh-MB) y bosque seco premontano (bs-PM) (Corporación Autónoma de Nariño [Corponariño], 2014).

Tabla 1. Datos de pasaporte de las localidades muestreadas en el municipio de Sandoná, departamento de Nariño.

\begin{tabular}{|c|c|c|c|c|c|c|}
\hline Finca & Zona & Coordenadas & $\begin{array}{l}\text { Altitud } \\
\text { (msnm) }\end{array}$ & $\begin{array}{c}\mathrm{T} \\
\left({ }^{\circ} \mathrm{C}\right)\end{array}$ & $\begin{array}{l}\text { HR } \\
(\%)\end{array}$ & $\begin{array}{c}\text { PP } \\
(\mathrm{mm} \text { año-1) }\end{array}$ \\
\hline La Cruz & $A$ & $\begin{array}{l}1^{\circ} 12^{\prime} 27^{\prime \prime} \\
77^{\circ} 46^{\prime} 45^{\prime \prime}\end{array}$ & 2.015 & 18 & 81,30 & $1.197,6$ \\
\hline EI Mana & $M$ & $\begin{array}{l}1^{\circ} 10^{\prime} 25^{\prime \prime} \\
77^{\circ} 48^{\prime} 32^{\prime \prime}\end{array}$ & 1.700 & 20 & 75,56 & 837,8 \\
\hline Las Delicias & $B$ & $\begin{array}{l}1^{\circ} 13^{\prime} 36^{\prime \prime} \\
77^{\circ} 44^{\prime} 54^{\prime \prime}\end{array}$ & 1.536 & 21 & 76,53 & $1.205,4$ \\
\hline
\end{tabular}

Fuente: autores de esta investigación. 


\section{Área experimental}

El trabajo se realizó en lotes experimentales del proyecto Investigación Evaluación del efecto de sombra de diferentes especies arbóreas en el comportamiento agronómico y calidad de café Consacá, Nariño, Occidente, financiado por el Sistema General de Regalías y ejecutado por el Grupo de Investigación en Producción de Frutales Andinos de la Universidad de Nariño. Los datos se obtuvieron de un sistema de café //Albizia carbonaria// Inga densiflora. En cada una de las tres altitudes se seleccionó una finca cafetera (Tabla 1) sembrada con café variedad Castillo ( 5 años de edad), con una distancia de siembra de 1,30 x 1,30 m al triangulo, en asociación con las especies leñosas (guamo, carbonero), sembradas a una distancia de $9 \times 9 \mathrm{~m}$; dentro de cada finca se tomó una parcela con un área de $2500 \mathrm{~m}^{2}$ donde se tomaron al azar cuatro plantas de café, las cuales fueron marcadas para, posteriormente, hacer sobre ellas el registro periódico de las diferentes variables evaluadas.

\section{Labores culturales}

Durante el periodo de evaluación se realizaron dos aplicaciones de fertilizante (febrero y octubre) con fuentes simples (Urea, DAP, $\mathrm{KCl}$ ), más elementos menores. El control de las plantas arvenses se realizó, en promedio, dos veces por año (marzo y noviembre) con la utilización de guadaña. Además, se hicieron aplicaciones localizadas de fungicidas para problemas fitosanitarios, principalmente de Phoma sp., y Cercospora sp.

\section{Variables evaluadas}

Variables climáticas: precipitación (PP), humedad relativa (HR), temperatura (T) y Radiación Fotosintéticamente Activa (RFA), registradas durante el periodo de evaluación por estaciones meteorológicas Spectrum Technologies Inc, modelo 2900ET Weather Station, ubicadas en cada lote experimental.

Variables morfológicas: altura de planta (AP), la medición se realizó con una cinta métrica desde la parte basal hasta el meristemo apical del tallo principal del árbol; número de ramas primarias productivas $(R P)$, se contó de forma manual el número de ramas por planta evaluada; diámetro basal de planta (DB), la medición se realizó con un pie de rey; número de hojas $(\mathrm{NH})$, se contó de forma manual el total de hojas por planta evaluada; longitud de ramas (LR), se midió con una cinta métrica; número de entrenudos (NE), se contaron de forma manual. Las variables LR y NE se midieron en seis ramas, dos por cada tercio de planta (alto, medio y bajo). También se estimó el área foliar (AF) mediante el modelo matemático estimado por el Grupo de Investigación en Producción de Frutales Andinos [GPFA] de La Universidad de Nariño (ecuación 1); así como el Índice de Área Foliar (IAF) y rendimiento (RTO) en t/ha.

\section{Ecuación 1:}

$A F=a+b x / 1+c x+d x^{2}$

Donde: $a=4,541 ; b=2,34, c=0,154 ; d=$ 0,0075 y $x=$ longitud promedia de seis hojas.

Una vez se determinó el área foliar promedio por hoja, se multiplicó por el número total de hojas por planta, para así obtener el área foliar por planta. Estimado el $A F$, se dividió entre el área del suelo que ocupa la planta y se obtuvo el índice de área foliar. Para el cálculo del rendimiento, previamente a la cosecha se tomó una muestra aleatoria de diez (10) plantas por unidad de estudio, se contó el número total de frutos por planta y se calculó el peso de fruto, a partir del promedio de una muestra de cien frutos tomada al azar en la parcela evaluada. La evaluación de la producción de café cereza [CPS] se determinó a partir de diferenciales de peso de muestras de $12 \mathrm{~kg}$ de café cereza, tomadas por cada localidad experimental. Las muestras de café cereza fueron sometidas a un proceso de beneficio tradicional (se secaron a libre exposición solar y se registró el peso cuando las muestras alcanzaron un grado promedio de humedad de $12 \%$ ) y, finalmente, a partir de los datos obtenidos se estimó la producción de CPS en kilogramos por hectárea.

\section{Análisis de la información}

Las variables de tipo morfológico y fisiológico se sometieron a un Análisis de Varianza (ANDEVA), usando el software XLSTAT y teniendo en cuenta el modelo estadístico (ecuación 2). 
Ecuación 2: $\quad Y_{i j}=\mu+R_{j}+A_{i}+E_{i j}$

En este modelo $Y_{i j}$ es la variable de respuesta en la repetición j-ésima, altura i-ésima, $\mu$ es el efecto de la media general, $R_{j}$ es el efecto de la repetición j-ésima, $A_{i}$ es el efecto de la altura i-ésima y $E_{i j}$ es el error asociado a la repetición j-ésima y altura i-ésima. En las variables donde se rechazó la hipótesis nula, se utilizó la prueba de comparación de medias de LSD Fisher. Posteriormente, en cada zona altitudinal se realizó un Análisis de Correlación múltiple de Pearson para establecer el nivel de similitud entre las variables morfológicas, fisiológicas y climáticas con el rendimiento. Con base en lo anterior, se seleccionó la variable que presento una correlación significativa para establecer las relaciones causa-efecto entre ésta y el resto. De esta forma, indirectamente, se está estimando el efecto de las variables sobre el rendimiento a través del Análisis de Sendero, el cual permite descomponer las correlaciones entre dos variables ( $X$ e $Y$ ) en: una suma del efecto directo de $X$ sobre $Y, y$ efectos indirectos de $X$ sobre $Y$ a través de otras variables independientes en un sistema de correlaciones. Este análisis tiene el objetivo de identificar las posibles explicaciones causales de las relaciones entre una variable respuesta (dependiente) y las variables independientes (Abbott et al., 2007).

\section{RESULTADOS Y DISCUSIÓN}

El ANDEVA (Tabla 2) indica que solo existieron diferencias significativas entre altitudes en las variables: altura de planta, temperatura ambiente, precipitación y radiación fotosintéticamente activa. Las variables número de hojas, diámetro basal, número de ramas primarias, largo de ramas, número de nudos y largo de hoja no presentaron diferencias estadísticas significativas.

Tabla 2. Cuadrados medios del Análisis de varianza para las variables morfológicas, climáticas y rendimiento, evaluadas en tres altitudes $(A=2.015 \mathrm{msnm}, M=1.700 \mathrm{msnm}, B=1.536 \mathrm{msnm})$ de una zona productora de café en el departamento de Nariño.

\begin{tabular}{lcccccccc}
\hline FV & GL & AP & NH & DB & RP & LR & NE & LF \\
Altitud & 2 & $\mathbf{3 4 2 6 , 7 5 *}$ & 63101,22 & 6,27 & 149,97 & 48,93 & 11,84 & 0,89 \\
Repetición 3 & $\mathbf{1 6 8 5 , 1 8 *}$ & 67229,94 & 3,10 & 97,91 & 22,89 & 6,59 & 1,76 \\
Error & 6 & 321,34 & 17689,69 & 2 & 64,93 & 87,90 & 8,82 & 0,78 \\
Media & & 88,81 & 831,79 & 9,54 & 61,68 & 50,19 & 17,21 & 24,77 \\
CV (\%) & & 20,18 & 15,99 & 14,83 & 13,06 & 18,68 & 17,26 & 3,55 \\
\hline FV & GL & AF & IAF & RTO & HR & T & PP & RFA \\
\hline Altitud & 2 & 191,09 & $1,1 \mathrm{E}-05$ & 3,59 & 7,14 & $\mathbf{2 , 0 2 * *}$ & $\mathbf{5 2 4 , 4 8 *}$ & $\mathbf{6 7 0 1 5 , 9 *}$ \\
Repetición 3 & 92,62 & $1,7 \mathrm{E}-05$ & $\mathbf{0 , 5 1 *}$ & 4,07 & 0,10 & 395,95 & 14235,63 \\
Error & 6 & 75,28 & $2,5 \mathrm{E}-05$ & 0,24 & 3,78 & 0,11 & 74,9 & 3850,81 \\
Media & & 114,61 & 0,01 & 2,73 & 77,92 & 76,99 & 65,66 & 461,315 \\
CV (\%) & 7,57 & 37,92 & 18,07 & 2,50 & 1,70 & 52,73 & 13,45 \\
\hline
\end{tabular}

*: Diferencias significativas $(\mathrm{P}<0.05) ; * *$ : Diferencias altamente significativas; $\mathrm{CV}$ : Coeficiente de variación; AP: Altura de planta; NH: Numero de hojas; DB: Diámetro basal; RP: Número de ramas primarias por planta; LR: Largo de ramas primarias; NE: Número de entrenudos de ramas primarias; LH: Largo de hoja; AF: Área foliar; IAF: Índice de área foliar; RFA: Radiación Fotosintéticamente Activa; RH: Humedad relativa; T: Temperatura; PP: Precipitación; RTO: Rendimiento.

Fuente: autores de esta investigación.

Según la prueba de comparación de medias (Tabla 3), el comportamiento de la AP en las zonas media y alta fue estadísticamente similar con promedios de 90,80 y $117,04 \mathrm{~cm}$ respectivamente, difiriendo con la altitud baja (1.536 msnm). La actividad fotosintética de la planta no solo depende del aumento o disminución de la temperatura sino también de factores como la disponibilidad hídrica en el suelo, que garantiza la eficiencia fotosintética y por ende el buen crecimiento y desarrollo de la planta, (Welker et al., 2004; Sanfuentes et al., 2012). 
El comportamiento de la RFA presentó diferencias significativas entre las tres zonas de estudio, en la altitud Baja (1.536 msnm) se obtuvo el promedio más alto, con 593,71 $\mu \mathrm{mol} . \mathrm{m}^{-2} . \mathrm{s}^{-2}$; seguida de la zona Media (1.700 msnm), con $455,17 \mu \mathrm{mol} . \mathrm{m}^{-2} \cdot \mathrm{s}^{-2}$; y la zona Alta (2.015msnm), con 335,06 $\mu \mathrm{mol} . \mathrm{m}^{-2} . \mathrm{s}^{-2}$ (Tabla 3). La RFA es una variable de gran importancia para las plantas, ya que se traduce en la regulación del crecimiento, desarrollo y productividad de las plantas (Liu et al., 2004; Bote et al., 2018). Es por esto que la variación de la luminosidad, en las diferentes zonas altitudinales, se encuentra asociada con las diferencias en la producción (Camayo-Vélez et al., 2003; Criollo et al., 2014).

Tabla 3. Comparación de medias (DMS) para las variables evaluadas en las diferentes alturas $(A=2.015 \mathrm{msnm}, \mathrm{M}$ $=1.700 \mathrm{msnm}, \mathrm{B}=1.536 \mathrm{msnm}$ ) de una zona productora de café en el departamento de Nariño.

\begin{tabular}{|c|c|c|c|c|c|c|c|c|c|c|c|c|}
\hline VARTABLE & AP & & NH & & DB & & $\mathbf{R P}$ & & LR & & NE & LH \\
\hline CRUZ & 117 & $A$ & 975,9 & A & 5,59 & $A$ & 66,13 & $A$ & 52,74 & $A$ & 18,35 & A $11,86 \mathrm{~A}$ \\
\hline MANA & 90,8 & $A$ & 745,9 & A & 4,67 & $A B$ & 66,95 & $A$ & 51,63 & $A$ & 18,06 & A $11,86 \mathrm{~A}$ \\
\hline DELICIAS & 58,6 & $B$ & 773,6 & A & 4,14 & B & 54,96 & $A$ & 46,2 & $A$ & 15,23 & $12,8 \quad A$ \\
\hline VARTABLE & $\mathbf{A F}$ & & IAF & & RTO & & RFA & & HR & & $\mathrm{T}$ & PP \\
\hline CRUZ & 121,5 & $A$ & 0,01 & A & 1,68 & B & 335,1 & $\mathrm{C}$ & 76,41 & $A$ & 18,48 & B 28,56 A \\
\hline MANA & 107,7 & $A$ & 0,01 & A & 3,52 & $A$ & 455,2 & $B$ & 78,94 & $A$ & 19,39 & A $\quad 5,81 \quad$ B \\
\hline DELICIAS & 114,7 & $A$ & 0,01 & $A$ & 2,99 & A & 593,7 & $A$ & 78,41 & $A$ & 19,88 & A $14,87 \mathrm{AB}$ \\
\hline
\end{tabular}

AP: Altura de planta; NH: Numero de hojas; DB: Diámetro basal; RP: Número de ramas primarias por planta; LR: Largo de ramas primarias; NE: Número de entrenudos de ramas primarias; LH: Largo de hoja; AF: Área foliar; IAF: Índice de área foliar; RFA: Radiación Fotosintéticamente Activa; RH: Humedad relativa; T: Temperatura; PP: Precipitación; RTO: Rendimiento. Medias con distintas letras son significativas a una $\mathrm{P}<0,05$.

Fuente: autores de esta investigación.

Las Tablas 4,5 y 6 corresponden a los análisis de sendero de las tres altitudes evaluadas, en las que se muestran las relaciones que existen entre la variable RTO y las variables morfológicas, fisiológicas y climáticas. En la zona alta se observó que las variables morfológicas que presentaron los mayores efectos directos sobre el rendimiento fueron las variables $\operatorname{NE}(0,54)$ y $\operatorname{AP}(0,76)$. Dentro de las variables climáticas, la que mostró mayor efecto directo sobre el RTO fue la HR. Dentro de los efectos indirectos, los más significativos fueron los causados por el NH, DB, LR, NE y AF a través de la AP (Tabla 4).

La altura de la planta es considerada como una variable que contribuye al desarrollo de ramas primarias y secundarias que garantizan la producción de una planta (Blanco et al., 2003; Balladares y Calero, 2005; Arcila et. al., 2007). El número de hojas aporta a la actividad fotosintética, puesto que entre mayor número de hojas aumenta la capacidad de interceptación de radiación solar y por ende, existe una mayor productividad (Barraza et al., 2004; Escalante y Rodríguez, 2010; Da Silva et al., 2011).

Una planta de café para el desarrollo de los sus frutos requiere hasta cuatro veces más fotoasimilados que los que necesita para la generación de una nueva rama, y se espera que a mayor número de ramas exista una mayor producción, lo cual depende de factores climáticos como la radiación solar (Vaast et al., 2005; Escalante y Rodríguez, 2010). El requerimiento de la humedad relativa en el cultivo de café esta entre 70$95 \%$ y es un factor importante, puesto que influye sobre el crecimiento de tejidos, transpiración de la planta, fecundación de las flores, entre otras (Fernández, 2018). 
Tabla 4. Análisis de sendero que muestra los efectos directos (diagonal) e indirectos (fuera de la diagonal) de los componentes morfológicos y climáticos sobre el rendimiento del cultivo de café en la altitud alta (2.015 msnm).

\begin{tabular}{lcccccccccccccc}
\hline & $A P$ & $N H$ & $D B$ & $R P$ & $L R$ & $N E$ & $L H$ & $A F$ & TAF & $R F A$ & $H R$ & $T$ & $P P$ & XX-RTO \\
AP & $\mathbf{0 , 7 6}$ & $-0,02$ & $-0,08$ & $-0,05$ & 0,12 & 0,43 & $-0,14$ & $-0,20$ & $-0,22$ & 0,04 & 0,13 & 0,03 & 0,16 & 0,95 \\
NH & 0,70 & $-\mathbf{0 , 0 2}$ & $-0,07$ & $-0,07$ & 0,08 & 0,26 & $-0,29$ & $-0,20$ & $-0,06$ & 0,08 & 0,26 & 0,07 & 0,24 & 0,98 \\
DB & 0,61 & $-0,02$ & $-\mathbf{0 , 1 0}$ & $-0,02$ & 0,09 & 0,36 & 0,12 & $-0,12$ & $-0,28$ & 0,09 & $-0,01$ & $-0,01$ & 0,14 & 0,85 \\
RP & 0,46 & $-0,02$ & $-0,03$ & $-\mathbf{0 , 0 8}$ & 0,02 & 0,01 & $-0,52$ & $-0,18$ & 0,19 & 0,07 & 0,41 & 0,12 & 0,27 & 0,71 \\
LR & 0,65 & $-0,01$ & $-0,06$ & $-0,01$ & $\mathbf{0 , 1 4}$ & 0,53 & 0,06 & $-0,15$ & $-0,35$ & $-0,02$ & $-0,07$ & $-0,03$ & 0,00 & 0,67 \\
NE & 0,60 & $-0,01$ & $-0,06$ & 0,00 & 0,13 & $\mathbf{0 , 5 4}$ & 0,17 & $-0,12$ & $-0,40$ & $-0,03$ & $-0,13$ & $-0,05$ & $-0,03$ & 0,60 \\
LH & $-0,18$ & 0,01 & $-0,02$ & 0,07 & 0,01 & 0,15 & $\mathbf{0 , 5 9}$ & 0,13 & $-0,32$ & $-0,02$ & $-0,42$ & $-0,13$ & $-0,20$ & $-0,31$ \\
AF & 0,69 & $-0,02$ & $-0,05$ & $-0,07$ & 0,09 & 0,30 & $-0,36$ & $-\mathbf{0 , 2 2}$ & $-0,05$ & 0,04 & 0,27 & 0,07 & 0,20 & 0,89 \\
RFA & $-0,22$ & 0,01 & 0,06 & 0,04 & 0,02 & 0,10 & 0,09 & 0,05 & $-0,09$ & $-\mathbf{0 , 1 4}$ & $-0,19$ & $-0,06$ & $-0,24$ & $-0,55$ \\
HR & 0,22 & $-0,01$ & 0,00 & $-0,08$ & $-0,02$ & $-0,17$ & $-0,57$ & $-0,14$ & 0,32 & 0,06 & $\mathbf{0 , 4 3}$ & 0,13 & 0,25 & 0,43 \\
PP & $-0,40$ & 0,02 & 0,05 & 0,07 & 0,00 & 0,05 & 0,39 & 0,15 & $-0,17$ & $-0,12$ & $-0,36$ & $-0,11$ & $-\mathbf{0 , 3 0}$ & $-0,73$ \\
\hline
\end{tabular}

AP: Altura de planta; NH: Numero de hojas; DB: Diámetro basal; RP: Número de ramas primarias por planta; LR: Largo de ramas primarias; NE: Número de entrenudos de ramas primarias; LH: Largo de hoja; AF: Área foliar; IAF: Índice de área foliar; RFA: Radiación Fotosintéticamente Activa; RH: Humedad relativa; T: Temperatura; PP: Precipitación; RTO: Rendimiento.

Fuente: autores de esta investigación.

En la Tabla 5 se presenta el Análisis de Sendero para la zona media (1.700 msnm), en la que se observa que la variable que presentó el mayor efecto directo fue RP $(0,73)$. Dentro de los efectos indirectos, los más importantes fueron los causados por RH $(0,73)$ a través de T. La arquitectura de la planta para la disposición de las ramas primarias indica buena posición de flores y mejor cuajado de frutos, los cuales son indicadores de un buen rendimiento (Quevedo et al., 2015). Sin embargo, a pesar de que el número de ramas primarias tiene un efecto directo sobre el rendimiento, presenta unos efectos indirectos negativos acumulativos a través del $\mathrm{NH}$, el LR y la HR. Hay que anotar que el aumento del número de ramas es una característica morfológica del café relacionada con la productividad (Balladares y Calero, 2005). Cada cultivo tiene diferentes requerimientos durante las diferentes etapas de desarrollo fenológico y entre más proximidad haya a los valores óptimos requeridos por el cultivo, su productividad será mayor (López-López y Benavides, 2014).

Tabla 5. Análisis de Sendero que muestra los efectos directos (diagonal) e indirectos (fuera de la diagonal), de los componentes morfológicos y climáticos sobre el rendimiento del cultivo de café a una altitud de $1.700 \mathrm{msnm}$.

\begin{tabular}{lcccccccccccccc|}
\hline V & $A P$ & $N H$ & DB & RP & LR & NE & LH & AF & IAF & RFA & HR & T & PP & RX-RTO \\
AP & 0,15 & 0,43 & 0,01 & $-0,65$ & 0,39 & $-0,02$ & 0,23 & $-0,22$ & 0,02 & 0,00 & 0,53 & $-0,07$ & $-0,33$ & 0,46 \\
RP & $-0,13$ & $-0,34$ & 0,00 & $\mathbf{0 , 7 3}$ & $-0,44$ & 0,03 & $-0,23$ & 0,03 & $-0,03$ & $-0,03$ & $-0,51$ & 0,07 & 0,24 & $-0,59$ \\
LH & $-0,09$ & 0,04 & $-0,01$ & 0,43 & $-0,04$ & $-0,02$ & $-\mathbf{0 , 3 9}$ & 0,07 & 0,00 & 0,00 & $-1,15$ & 0,06 & 0,14 & $-0,96$ \\
HR & $-0,07$ & 0,13 & $-0,01$ & 0,32 & 0,05 & $-0,03$ & $-0,38$ & 0,06 & 0,01 & 0,00 & $\mathbf{- 1 , 1 7}$ & 0,05 & 0,10 & $-0,94$ \\
T & 0,13 & 0,27 & 0,00 & $-0,71$ & 0,37 & $-0,02$ & 0,29 & $-0,05$ & 0,02 & 0,02 & $\mathbf{0 , 7 3}$ & $\mathbf{- 0 , 0 8}$ & $-0,24$ & 0,74
\end{tabular}

V: Variable; AP: Altura de planta; NH: Numero de hojas; DB: Diámetro basal; RP: Número de ramas primarias por planta; LR: Largo de ramas primarias; NE: Número de entrenudos de ramas primarias; LH: Largo de hoja; AF: Área foliar; IAF: Índice de área foliar; RFA: Radiación Fotosintéticamente Activa; RH: Humedad relativa; T: Temperatura; PP: Precipitación; RTO: Rendimiento.

Fuente: autores de esta investigación. 
El análisis de sendero para la zona Baja (1.536 msnm) mostró que las variables que presentaron los mayores efectos directos sobre el RTO de café fueron LR y el AF, y dentro de las variables climáticas fue la T. Los efectos indirectos más importantes fueron el NE a través de la AP $(0,80)$, el DB a través del $\mathrm{NH}(0,72)$, RP a través de $\mathrm{LH}$, AF y HR. Estos efectos fueron determinados por las condiciones climáticas de la zona (Tabla 6). Por otra parte, los factores que regulan el crecimiento y la productividad del café están asociados a la radiación solar, su interceptación y su uso eficiente (Montoya et al., 2009). Una mayor área foliar implica, generalmente, una mayor capacidad de intercepción de radiación solar y por ende una mayor productividad (Da Silva et al., 2011) y, como lo manifiesta Pérez et al. (2013) un mayor número de ramas y mayor número de entrenudos son indicadores de mayor rendimiento, lo cual coincide con las relaciones causa-efecto encontradas en esta altitud de estudio.

Tabla 6. Análisis de sendero que muestra los efectos directos (diagonal) e indirectos (fuera de la diagonal) de los componentes morfológicos y climáticos del rendimiento del cultivo de café a una altitud de $1.536 \mathrm{msnm}$.

\begin{tabular}{lccccccccccccccc}
\hline V & $A P$ & $N H$ & $D B$ & $R P$ & $L R$ & $N E$ & $L H$ & $A F$ & IAF & $R F A$ & $H R$ & $T$ & $P P$ & $R X-R T 0$ \\
DB & $-1,39$ & 0,72 & $\mathbf{0 , 4 2}$ & $-0,26$ & 0,04 & 0,06 & $-0,07$ & $-0,12$ & $-0,19$ & 0,53 & 0,62 & 0,45 & $-0,43$ & 0,40 \\
RP & 0,07 & $-0,02$ & 0,16 & $-\mathbf{0 , 7 0}$ & 0,35 & $-0,87$ & 0,52 & 0,62 & 0,12 & $-0,64$ & 1,13 & 0,23 & $-0,04$ & 0,95 \\
LR & 0,84 & $-0,18$ & 0,05 & $-0,60$ & $\mathbf{0 , 4 1}$ & $-0,87$ & 0,45 & 0,57 & 0,30 & $-1,23$ & 0,92 & $-0,03$ & 0,03 & 0,66 \\
NE & 0,80 & $-0,35$ & $-0,03$ & $-0,63$ & 0,37 & $-\mathbf{0 , 9 7}$ & 0,58 & 0,72 & 0,23 & $-1,02$ & 0,94 & 0,02 & 0,15 & 0,81 \\
LH & $-0,59$ & 0,39 & 0,05 & 0,60 & $-0,30$ & 0,93 & $-\mathbf{0 , 6 1}$ & $-0,74$ & $-0,15$ & 0,71 & $-0,88$ & $-0,08$ & $-0,19$ & $-0,86$ \\
AF & 0,71 & $-0,42$ & $-0,07$ & $-0,58$ & 0,31 & $-0,94$ & 0,61 & $\mathbf{0 , 7 4}$ & 0,17 & $-0,80$ & 0,84 & 0,04 & 0,21 & 0,83 \\
RH & 0,20 & $-0,13$ & $-0,23$ & 0,68 & $-0,33$ & 0,78 & $-0,46$ & $-0,54$ & $-0,07$ & 0,49 & $\mathbf{- 1 , 1 6}$ & $-0,30$ & 0,12 & $-0,94$ \\
T & $-1,57$ & 0,53 & 0,35 & $-0,30$ & $-0,02$ & $-0,03$ & 0,09 & 0,06 & $-0,30$ & 0,90 & 0,64 & $\mathbf{0 , 5 5}$ & $-0,28$ & 0,61
\end{tabular}

V: Variable; AP: Altura de planta; NH: Numero de hojas; DB: Diámetro basal; RP: Número de ramas primarias por planta; LR: Largo de ramas primarias; NE: Número de entrenudos de ramas primarias; LH: Largo de hoja; AF: Área foliar; IAF: Índice de área foliar; RFA: Radiación Fotosintéticamente Activa; RH: Humedad relativa; T: Temperatura; PP: Precipitación; RTO: Rendimiento.

Fuente: autores de esta investigación.

\section{CONCLUSIONES}

Los componentes de rendimiento del café (variedad Castillo) cambiaron con la altitud. Las zonas Media y Baja obtuvieron los mayores rendimientos. Para la zona Media (1.700 msnm), los efectos directos sobre el RTO fueron ocasionados por la variable RP. Dentro de los efectos indirectos, los más importantes fueron los causados por la variable RH a través de la T. En la zona Baja (1.536 msnm), los mayores efectos directos sobre el RTO los provocaron las variables LR,
AF y RFA; y los efectos indirectos fueron la $T$ a través de la RFA, el NE a través de la AP, el DB a través del NH y el RP a través de la longitud de la hoja (LH), del área foliar (AF) y de la humedad relativa (HR). Finalmente, la zona que presentó el menor rendimiento fue la zona Alta (2.015 msnm), mostrando comoefectos directos la afectación en las variables $A P, N E$ y $H R$; y los indirectos, $N H$, $D B, L R, A F$ a través de la AP. Se hace necesario estudiar la arquitectura de la planta y su relación con la HR para potenciar el RTO del café. 


\section{CONTRIBUCIÓN DE LA AUTORÍA}

Paula Yulitza Burbano R.: registro de datos, sistematización de datos, análisis de información, revisión bibliográfica y redacción del artículo.

Angie Lorena Valencia A.: registro de datos, sistematización de datos, análisis de información, revisión bibliográfica y redacción del artículo.

Tulio César Lagos-Burbano: Coordinación del estudio, análisis de datos, estadística, revisión y corrección del artículo.

\section{AGRADECIMIENTOS}

Los autores expresan sus agradecimientos al Sistema General de Regalías, a la Facultad de Ciencias Agrícolas de la Universidad de Nariño, a través del Grupo de Investigación en Producción de Frutales Andinos y al proyecto Investigación evaluación del efecto de sombra de diferentes especies arbóreas en el comportamiento agronómico y calidad de Café, Consacá, Nariño, Occidente, Código BPIN (2012000100184); y en especial a quien, en vida, fue el PhD. Hernando Criollo Escobar, por sus aportes en la formulación y dedicación en el desarrollo del presente estudio.

\section{LITERATURA CITADA}

Abbott, L. A., Pistorale, S. M. y Filippini, O (2007). Análisis de coeficientes de sendero para el rendimiento de semillas en Bromus catharticus. Ciencia e investigación agraria, 34(2), 141-149. http://dx.doi.org/10.4067/ S0718-16202007000200007

Arcila, P. (2007). Densidad de siembra y productividad de los cafetales. En J. P. Arcila, F. V. Farfán, L. A. Moreno, L. F. Salazar y E. Hincapié (Eds.). Sistemas de producción de café en Colombia (pp. 132-143). Cenicafé.

Balladares, D. y Calero, J. (2005). Efecto de la sombra y fertilización sobre el crecimiento, estructura productiva y calidad de café (Coffea arabica) vr. Costa Rica 95 [Tesis de pregrado, Universidad Nacional Agraria]. Repositorio institucional. https:// repositorio. una.edu.ni/1969/1/tnf04b188. pdf

Barraza, F., Fischer, G. y Cardona, C. (2004). Estudio del proceso de crecimiento del cultivo del tomate (Lycopersicon esculentum Mill.) en el Valle del Sinú medio, Colombia. Agronomía Colombiana, 22(1), 81-90. https://www.redalyc.org/ pdf/1803/180317823011.pdf

Blanco, M., Haggar, J., Morada, P., Madriz, J. y Pavón, G. (2003). Morfología del café (Coffea arabica L.), en lotes comerciales. Nicaragua. Agronomía Mesoamericana, 14(1), 97-103. https://dialnet.unirioja.es/ descarga/articulo/5546834.pdf

Bote, A., Ayalew, B., Ocho, F., Anten, N. \& Vos, J. (2018). Analysis of coffee (Cofea arabica L.) performance in relation to radiation levels and rates of nitrogen supply I. Vegetative growth, production and distribution of biomass and radiation use efficiency. European Journal of Agronomy. 92, 115-122. http://dx.doi.org/10.1016/j. eja.2017.10.007

Camayo-Vélez, G. C., Chaves-Córdoba, B., Arcila-Pulgarín, J. y JaramilloRobledo, Á. (2003). Desarrollo floral del cafeto y su relación con las condiciones climáticas de Chinchiná - Caldas. Cenicafé, 54(1), 35-49.https://biblioteca. cenicafe.org/bitstream/10778/264/1/ arc054\%2801\%29035-049.pdf

Castro, N., Domínguez, R. y Paccapelo, H. (2011). Análisis del rendimiento de grano y sus componentes en cereales sintéticos. Semiárida, 22, 13-21. http://www. biblioteca.unlpam.edu.ar/pubpdf/rev-agro/ v22a03castro.pdf

Checa, J. E. y Noguera, W. A. (2017). Oferta ambiental, crecimiento inicial del café (Coffea arabica L) en la zona cafetera de Nariño [Tesis de pregrado, Universidad de Nariño]. Archivo digital. http://sired.udenar.edu. co/5953/1/OFERTA\%20AMBIENTAL\%2C\%20 CRECIMIENTO \%20INICIAL \% 20DEL \% 20 CAF\%C3\%89\%20\%28COFFEA\%20ARABI. pdf

Clavijo, S. (2019, 12 de octubre). Panorama cafetero 2019-2020. La República. https:// www.google.com.co/amp/s/amp.larepublica. co/analisis/sergio-clavijo-500041/panoramacafetero-2019-2020-2920631 
Comité Departamental de Cafeteros de Nariño. (2008). Informe del Comité Departamental de Cafeteros de Nariño. https://federaciondecafeteros.org/static/ files/Nariño4.pdf

Corporación Autónoma Regional de Nariño. (2014). Diagnóstico Biofísico y Socioeconómico Municipio de Consacá. https://corponarino.gov.co/expedientes/ intervencion / D I A G N OSTICO \% 20 B I O F I S I C O \% 20 Y \% 20 S O C I O \% 20 ECONOMICO\%20DE\%20CONSACA.pdf

Criollo, H., Lagos, T. C., Fischer, G., Mora, L., y Zamudio, L. (2014). Comportamiento de tres genotipos de uchuva (Physalis. peruviana L.) bajo diferentes sistemas de poda. Revista Colombiana de Ciencias Hortícolas. 8(1), 3443. http://www.scielo.org.co/pdf/rcch/v8n1/ v8n1a04.pdf

Criollo, H., Benavides, D., Muñoz, J. y Lagos, T. C. (2019). Caracterización socioeconómica de fincas cafeteras del departamento de Nariño, Colombia. Revista. U.D.C.A: Actualidad \& Divulgación Científica, 22(2), 1-8. https://doi.org/10.31910/rudca. v22.n2.2019.1397

Da Silva, W., Batista, S., Tomaz, M. A., Teixeira, J. F., Nunes, W. y Deleon, L. (2011). Métodos de estimativa de área foliar em cafeeiro. Enciclopédia Biosfera. 7(13),746759. https://conhecer.org.br/ojs/index.php/ biosfera/article/view/4161/4050

Escalante, J. y Rodríguez, M. (2010). Biomasa, índice de cosecha y componentes del rendimiento en frijol y nitrógeno. Ciencias Agrícolas Informa, 19, 5-11.

Jaramillo-Robledo, A. (2005). Clima andino y café en Colombia. Cenicafé. https://biblioteca.cenicafe.org/ bitstream/10778/859/1/Portada.pdf

Liu, J., Miller, J. R., Pattey, E., Haboudane, D., Strachan, I. B. \& Hinther, M. (2004, del 20 al 24 de septiembre). Monitoring crop biomass accumulation using multi-temporal hyperspectral sensing data [conferencia]. IEEE International Geoscience and Remote Sensing Symposium, Anchorage, Estados Unidos. https://doi.org/10.1109/ IGARSS. 2004.1370643
López-López, A. J., y Benavides-León, C. (2014) Respuesta térmica del invernadero de la estación experimental Fabio Baudrit Moreno, Alajuela, Costa Rica. Agronomía Mesoamericana. 25(1), 121-132. https:// www.scielo.sa.cr/scielo.php?script $=$ sci arttext\&pid=S1659-13212014000100012

Ministerio de Agricultura y Desarrollo Rural. (2020). Cadena Café: $1^{\circ}$ Trimestre $2020 . \quad$ https://sioc.minagricultura.gov. co/Cafe/Documentos/2020-03-31\%20 Cifras\%20Sectoriales.pdf

Montoya, E. C., Arcila, J., Jaramillo, A., Riaño, N. y Quiroga, F. ( 2009). Modelo para simular la producción potencial del cultivo de café en Colombia. Cenicafé. https://www. cenicafe.org/es/publications/bot033.pdf Catie.

Muschler, R. (1999). Árboles en cafetales.

Organización Internacional del Café. (2019). Informe de la OIC sobre desarrollo cafetero del 2019. http://www.ico.org/ documents/cy2018-19/ed-2318c-overviewflagship-report.pdf

Pérez, E., Medrano, L. y Sánchez, J. (2013). El Path Analysis: Conceptos básicos y ejemplos de aplicación. Revista Argentina de Ciencias del Comportamiento. 5(1), 52-66. https://www.redalyc.org/ pdf/3334/333427385008.pdf

Quevedo, E., Sánchez, O. y Veloza, C. (2015). Efecto del tutorado y distancias de siembra sobre el rendimiento de Physalis peruviana L. Revista U.D.C.A: Actualidad \& Divulgación Científica. 18(1), 91-99. https://repository.udca.edu.co/ bitstream/11158/1599/1/Art\%C3\%ADculo. pdf

Ramírez, V. H., Peña, A. J., Jaramillo, A., Giraldo, J. P., Suárez, H. E. y Duque, N. (2012). Riesgo agroclimático para zona cafetera colombiana: método para regionalizar la variabilidad climática. Cenicafé. 63(2), 98-115. https://biblioteca.cenicafe.org/ handle/10778/539

Sanfuentes, C., Sierra-Almeida, A. y Cavieres, L. A. (2012). Efecto del aumento 
de la temperatura en la fotosíntesis de una especie alto-andina en dos altitudes. Gayana Botánica. 69(1), 37-45. http://dx.doi. org/10.4067/S0717-66432012000100005

Suárez, J. C., Rodríguez, E. y Duran, E. H. (2015). Efecto de las condiciones de cultivo, las características químicas del suelo y el manejo de grano en los atributos sensoriales de café (Cofea arabica L.) en taza. Acta Agronómica. 64(4), 342-348. http://dx.doi. org/10.15446/acag.v64n4.44641

Tirado, M., Tirado, L. y Mendoza, J. (2018). Interacción genotipo x ambiente en rendimiento de papa (Solanum tuberosum L.) con pulpa pigmentada en Cutervo, Perú. Chilean journal of agricultural \& animal sciences. 34(3), 191-198. https://dx.doi. org/10.4067/S0719-38902018005000502

Vaast, P., Angrand, J., Franck, N., Dauzat, J. \& Génard, M. (2005). Fruit load and branch ring-barking affect carbon allocation and photosynthesis of leaf and fruit of Cofea arabica in the field. Tree Physiology, 25(6), 753-760. https://doi.org/10.1093/ treephys/25.6.753
Wardofa, G. A., Asnake, D. \& Mohammed, H. (2019). Gge biplot analysis of genotype by environment interaction and grain yield stability of bread wheat genotypes in central ethiopia. Revista Journal of plant breeding and genetics. 7(2), 75-85. https://doi. org/10.33687/pbg.007.02.2846

Welker, J. M., Fahnestock, J. C., Henry, G. H. R., O'dea, K. W. \& Chimner, R. A. (2004). CO2 exchange in three Canadian High Arctic ecosystems: response to longterm experimental warming. Global Change Biology. 10(12), 1981-1995. https://doi. org/10.1111/j.1365-2486.2004.00857.x

Zapata, P. C., Andrade, H. J. y Nieto, Z. K. (2017). Comportamiento ecofisiológico del cafeto (Cofea arabica L.) CV. Castillo en sistemas agroforestales de Tibacuy, Cundinamarca. Revista. U.D.C.A: Actualidad \& Divulgación Científica. 20(1), 61-70. http://www.scielo.org.co/pdf/rudca/v20n1/ v20n1a08.pdf
Conflicto de intereses

Los autores declaran no tener ningún conflicto de intereses.

\section{(6) (1)(9)}

